\title{
José Luis Valdez Medina. Contribuciones actuales a la Psicología
}

\section{José Luis Valdez Medina. Current contributions to Psychology}

Norma Ivonne González Arratia López Fuentes

Sergio González Escobar

Yessica Paola Aguilar Montes de Oca

Marta Torres Muñoz

Jorge Alvarado Orozco

Universidad Autónoma del Estado de México

José Luis Valdez Medina nació un 7 de enero en la Ciudad de México y siempre mostró gran curiosidad por todo lo que les sucedía a los individuos. Desde su juventud se interesó por el comportamiento humano. Con tal interés, cursó la Licenciatura, Maestría y Doctorado en Psicología en la Universidad Nacional Autónoma de México (UNAM), donde obtuvo menciones honoríficas, incluso recibió la Medalla Gabino Barrera, otorgada por la UNAM a nivel doctoral. Además, se hizo acreedor al Premio Estatal de Ciencia y Tecnología (COMECYT), concedido por el Gobierno del Estado de México. Fue Miembro del Sistema Nacional de Investigadores (SNI), Nivel II, un hito en la psicología, incansable e insustituible miembro del Cuerpo Académico Consolidado Cultura y Personalidad. El mismo aludía a la fortuna que tuvo al ser alumno de brillantes y reconocidos Investigadores tanto a nivel nacional como Internacional, como el Dr. Jesús Figueroa Nazumo, Dr. Rogelio Díaz Guerrero, Dra. Isabel Reyes Lagunes, y Dr. Rolando Díaz Loving, quienes no sólo influyeron de manera profunda en su formación profesional, sino que también lo impulsaron; él siempre lo dijo "ellos son mis modelos a

Nota del autor

Norma Ivonne González Arratia López Fuentes, Facultad de Ciencias de la Conducta, Universidad Autónoma del Estado de México (UAEM); Sergio González Escobar, Facultad de Ciencias de la Conducta, UAEM; Yessica Paola Aguilar Montes de Oca, Facultad de Ciencias de la Conducta, UAEM; Martha Torres Muñoz, Facultad de Ciencias de la Conducta, UAEM; Jorge Alvarado Orozco, Facultad de Ciencias de la Conducta, UAEM.

La correspondencia en relación con este artículo debe dirigirse a Norma Ivonne González Arratia López Fuentes, Facultad de Ciencias de la Conducta, UAEM, Filiberto Gómez S/N, Km. 1.5 TolucaNaucalpan, Col. Guadalupe, C.P. 50010, Toluca, Edo. de México.

Dirección electrónica: nigalf@yahoo.com.mx 
seguir". Doctores que siempre le brindaron sus consejos, apoyo y cariño: Dra. Patricia Andrade Palos, Dra. Sofía Rivera Aragón, Dra. Roxana Sánchez Aragón, Dra. Mirta Flores Galaz, Dra. Inés Vargas, Dra. Tania Rocha Sánchez, Dra. Teresita Castillo, y muchos, muchos más.

A lo largo del desarrollo de la investigación en psicología, José Luis Valdez fue aproximándose al estudio de la conducta e hizo de él su principal objeto de estudio; sus avances consistieron en explicar la conducta como un fenómeno que tiene su origen en la construcción y reconstrucción del mundo a nivel mental. Los teóricos han explicado que dicho conocimiento se va agrupando a nivel de la memoria, en forma de mapas cognitivos o redes de información, que incluso pueden ser infinitas, ya que dependen de la cantidad de conocimientos que se vayan acumulando, generando significados psicológicos, a partir de los cuales se da el comportamiento de los individuos (Valdez, 1998). Tal conclusión significó para Valdez-Medina el inicio de una gran cantidad de estudios sobre el significado psicológico y, sobre todo, el uso de las Redes Semánticas Naturales como la técnica más relevante de acceder a redes de información.

A la fecha existe una gran cantidad de trabajos que destaca la variedad de posibilidades en cuanto a los usos y aplicaciones de la conducta. Su versatilidad se ha hecho patente en la gran diversidad de trabajos que indagaron sobre el significado psicológico de familia, bienestar, sexo, felicidad, maestro, partidos políticos, pareja, celos, apatía, vergüenza, salud, violen- cia, meta, logro, éxito, perdón, soledad, rico, pobre, virgen, madre, entre muchos otros conceptos e ideas.

Una de sus importantes contribuciones al conocimiento científico fue el impulso de las redes semánticas para el desarrollo de instrumentos psicológicos. Un ejemplo de ello es la evaluación del autoconcepto de Valdez y Reyes Lagunes, quienes mostraron en sus investigaciones que la mayoría de las mujeres continúan definiéndose como sentimentales, cariñosas, románticas y menos inteligentes en comparación con los hombres, lo cual denota rasgos de nuestra cultura, que incluso son bien valorados socialmente. Todos los estudios realizados por dichos autores, con muestran heterogéneas: niños hasta adultos, llevaron a Valdez Medina a considerar que las posibles diferencias en la percepción que tienen los individuos de sí mismos, se deben a un efecto de la cultura. Así, sus estudios con muestras de niños de diferentes países (España, Francia, Argentina, Chile y Brasil), nos proveen de amplia evidencia empírica para afirmar que la cultura juega un importante papel en el autoconcepto y la personalidad en general.

El crecimiento de las redes semánticas lo hizo considerar insuficiente indagar una sola palabra estímulo, por consiguiente, observó cómo era viable la contextualización de tal estímulo y propuso se hicieran frases estímulo, por ejemplo, además de definir la palabra "familia", se pueden incluir estímulos como: "para mí la familia es", "me gustaría que mi familia fuera". Todo ello permite ampliar la información para llegar a 
entender los mecanismos sociales involucrados. No basta sólo trabajar con el conjunto SAM, es posible establecer dimensiones con la finalidad de evitar perder información relevante. Tales dimensiones fueron llamadas "semánticas", y su estudio continua en boga hoy en día.

Gracias a su constancia y entusiasmo en la docencia e investigación, llegó a centrar su análisis en la temática de las diferencias entre los hombres y las mujeres (Valdez, DíazLoving \& Pérez, 2006). En esta obra se plasma una serie de estudios que, más allá de las diferencias entre lo masculino y lo femenino, resalta la complementariedad que también conlleva la interacción entre los géneros. Desde ésta perspectiva, los autores hacen una amplia revisión bibliográfica; los estudios que incluyen se destacan por su rigurosidad científica, y nos hacen reflexionar sobre la necesidad de integrar el aspecto biológico, el psicológico y el histórico sociocultural a las áreas de estudio para el entendimiento de los hombres y de las mujeres. Entre sus resultados, nos refieren que se van gestando cambios orientados a ver a la mujer como profesionista, inteligente, esposa, líder y con capacidad de compartir; aunque también continúan presentes algunos estereotipos orientados a la mujer sumisa, abnegada y menos inteligente que los hombres, a sabiendas de que cuentan con los medios necesarios para demostrar lo contrario (Valdez, 1999). Estos hallazgos sirvieron de punto de partida para entender las relaciones de pareja. En tal contexto, concluyeron que existe mayor discrepancia entre la realidad y el ideal en la elección de pareja, ya que hombres y mujeres eligen a su pareja en función de distintas características. En esta importante obra se incluye un estudio sobre las manifestaciones del amor en la pareja, la primera vez, lo mejor y lo peor de la relación en pareja, los celos, la infidelidad y el rompimiento con la pareja, así los autores concluyeron que es necesario esperar, para saber hacia dónde se moverán las ideas, los significados $\mathrm{y}$, por tanto, los comportamientos y el sistema de creencias sobre los hombres y las mujeres contemporáneos (Valdez et al., 2006).

Importantes interrogantes se hacía el Dr. Valdez Media respecto al comportamiento humano. Conforme pasaron los años y con la experiencia acumulada se planteó una serie de hipótesis acerca de la propia existencia para tratar de determinar cuál es el origen de todo el comportamiento. Sus lecturas de los filósofos clásicos y contemporáneos, teorías en psicología, e incluso sus estudios en el origen de las especies, dieron lugar a la propuesta de la Teoría de la Paz o Equilibrio (Valdez, 2009). La mencionada teoría que explica que el principio por excelencia que guía $\mathrm{u}$ orienta todo nuestro comportamiento es la paz o equilibrio, con su debida contraparte, el miedo. Este principio rector de su teoría, representa una relevante aportación a la Psicología, dado que integra tanto el aspecto biológico como el psicosociocultural, en el cual los organismos tendemos a buscar un estado de paz o equilibrio, tranquilidad, seguridad, protección, confianza, estabilidad; homeostasis que en la medida de lo posible nos mantenga libres de miedo, sobresaltos, amenazas, dolores o sufrimiento. Con base 
en lo anterior, se pretende que el individuo logre sobrevivir de forma exitosa en el ámbito individual, de grupos de referencia o a nivel especie.

Las estrategias comportamentales de origen natural o biológico, en las cuales nos vemos inmersos indican los parámetros de competencia, comparación, aceptación y rechazo. Ser competitivo, atractivo y tener buena jerarquía o estatus es insuficiente, si no somos aceptados o elegidos y no rechazados, por nosotros mismos y por los demás.

Sus premisas básicas son:

1. La vida es un proceso de transformación y adaptación que se auto-organiza.

2. Todos vivimos la vida entre el nacimiento y la muerte.

3. La muerte no es un estado de paz total, ni de movimiento, es sólo una más de las transformaciones o de los cambios o formas de adaptación, que la vida requiere para preservarse.

4. Toda la conducta se orienta por la conservación de la vida y la evitación de la muerte.

5. Se vive con base en dualidades que nos llevan a buscar un extremo y a evitar otro.

6. Toda la conducta parte de la detección que hacemos de nuestras necesidades o carencias y de nuestros miedos.

7. El principio o punto de partida y de llegada que guía toda nuestra conducta es el de la búsqueda de un estado de equilibrio, homeostasis, estabilidad que como contraparte nos lleva a evitar el miedo.

8. La paz, el equilibrio no son falta de movimiento.
9. El miedo es uno de los más importantes motores de la vida, puesto que es definido como la respuesta que da cualquier organismo vivo al intentar restablecer el estado de paz o equilibrio que se ha perdido o que se encuentra en riesgo de perderse. Con base en ello, puede decirse que todo se mueve desde el miedo.

10. Los seres humanos tenemos carencias o necesidades y miedos biológicos y socioculturales que nos llevan a buscar los recursos o satisfactores para encubrirlos.

11. Todos los seres humanos nos constituimos de dos aspectos elementales, centrales: el biológico, que nos lleva a buscar sobrevivir para intentar llegar al apareamiento con fines reproductivos y de conservación de la especie; así como el psicosociocultural, que nos orienta a buscar vivir, pero para vivir bien, $\mathrm{y}$ mejor que antes, que nosotros mismos y que los demás, es decir, nos incita a buscar jerarquía o estatus para intentar "llegar a ser alguien en la vida".

12. Todas las necesidades creadas se conectan directamente o tienen su origen en las necesidades de origen biológico.

13. Todos experimentamos en algún momento de nuestras vidas cualesquiera de los tipos de miedo (a la muerte, la enfermedad, el castigo, la venganza, la soledad, el abandono, el rechazo), que generan una reacción en el organismo, orientada a buscar un nuevo estado de estabilidad, puede decirse que todo se mueve desde el miedo y que se orienta a la paz o equilibrio. 
14. Todas las necesidades o carencias requieren de ser satisfechas para vivir en paz.

15. Todas las personas, debido a nuestra singularidad, tenemos necesidades o carencias muy específicas.

16. Todos evaluamos nuestras fortalezas y debilidades biológicas y psicosocioculturales a partir del resultado que obtenemos de nuestra participación en las estrategias comportamentales.

17. El objetivo psicosociocultural lleva a tolerar y controlar (fingir, aceptar, mentir, ocultar) y no aceptar y rechazar abiertamente. Con este tipo de situación de vida, se tiende a construir y desarrollar un tipo de personalidad débil o de ego (+) que busca la admiración, o de ego (-) que busca la compasión.

18. Toda aceptación favorable, ya sea en plano individual o social, nos mueve a rechazar o aceptar abiertamente; nos reintegra al círculo de la paz.

19. Por el contrario, toda evaluación desfavorable, cuando no se es capaz de aceptar o rechazar abiertamente y sin conflictos, comúnmente lleva a tolerar y a controlar. Por eso, se comienza a mentir, a engañar, a ocultar debilidades, aparentando o fingiendo ser diferentes de como se es realmente.

20. Todos buscamos un ideal de vida, por eso, difícilmente aceptamos nuestra forma de ser, de pensar, de hacer, de sentir. Así comienzan las tolerancias y los controles, el rechazo de nosotros mismos, porque pensamos que debemos modificarnos, ser mejores.

21. Todo tiene una causa y un efecto.
22. Se sigue la ley de la parsimonia, según la cual la explicación más simple es la mejor que hay.

Todos estos supuestos tienen valiosas implicaciones en el estudio contemporáneo de la Psicología, ya que proponen una visión integradora, coherente y congruente; ahora contamos con una teoría amplia para la explicación de la compleja conducta humana. Para tal propósito, se necesita tener una visión holística, que a su vez nos lleve no solo a evaluar las tendencias de personalidad, sino al mismo tiempo, nos ofrezca la posibilidad de un autoanálisis con el fin de orientarnos hacia un mejor nivel de salud mental, y por consiguiente nos dirija hacia la vida en equilibrio.

Como toda teoría, la Teoría de la Paz o Equilibrio requería de ser puesta a prueba con evidencia empírica, de hecho, Valdez Medina lo consideróunreto científico, probarestapropuesta, de ahí que ha habido importantes avances en dicho sentido. A manera de ejemplo, Valdez Medina responde a la pregunta ¿cómo se llega a la ansiedad, la depresión, a la desesperanza? Nos dice que haber experimentado y no resuelto alguna de las emociones negativas (frustración, odio, culpa, vergüenza, envidia, y celosegoísmo), se asocia con la presencia de niveles de ansiedad y manifestaciones depresivas.

Además de los principios de la teoría, en su libro (Valdez-Medina, 2009), nos ofrece una amplia explicación acerca de la dinámica y las consecuencias de vivir en el círculo del miedo, o en el de la paz o equilibrio, la dinámica de la envidia y los celos-egoismo, la dinámica del amor, 
el odio, el apego y la indiferencia. Y además nos ofrece su propuesta de evaluación de las tendencias de personalidad.

A la fecha, existen estudios que se han ocupado de investigar cada uno de los principios que sin duda corroboran la dinámica de la aceptación y el rechazo, la tolerancia y el control, la salud y la enfermedad. En este breve recorrido por la extensa obra del autor, esperamos haber podido captar lo sustancial de la Teoría de la Paz o Equilibrio. De ahí que hacemos una invitación para hacer una revisión más profunda de la misma en su libro titulado "Teoría de la Paz o Equilibrio", el cual está escrito en lenguaje sencillo, sin perder la profundidad ni la lógica que le subyace, ya que introduce al lector en un diálogo para mirar a otros, sobre todo, a sí mismo.

$\mathrm{Su}$ teoría resulta promisoria, aunque sin duda requiere de continuar investigando para contar con mayor evidencia de todos y cada uno de sus principios, lo cual nos abre un abanico de posibilidades para la mejor comprensión de los individuos. Así, en todos y cada uno de sus escritos, participaciones en congresos y foros académicos, entrevistas, dirección de tesis, docencia e investigación por más de 25 años, han dejado su huella, no sólo en nuestra Universidad, ha trascendido fronteras, y sin duda será referencia obligada en la investigación psicológica.

Afortunadamente, y desde su perspectiva, siempre consideró que "lo esencial es que cada uno de nosotros se haga cargo o responsable de sí mismo, puesto que aquél que llega a hacerse totalmente responsable de sí mismo, vive en paz o equilibrio, no se queja, está contento, estable, auto-organizado, con el menor desgaste posible. En otras palabras, quien se hace responsable de sí mismo, es alguien que se acepta abiertamente, $\mathrm{y}$ que vive, hace $\mathrm{y}$ es con $\mathrm{y}$ por gusto, $\mathrm{y}$ sin queja."

Desde ahora, el Dr. Valdez Medina nos sigue hablando a través de su obra y de todo su legado; su profundo conocimiento del ser humano, lo llevó a ser un gran maestro, excelente investigador, un asiduo colaborador, un compañero y amigo, cuya presencia y vigencia será siempre patente en la Psicología y en toda nuestra comunidad científica. Gracias por guiarnos en el camino de la investigación y generosidad al compartir tus conocimientos. Gracias por todas tus enseñanzas. ¡Hasta siempre!

\section{Referencias}

Valdez-Medina, J. L., Díaz-Loving, R., \& Pérez, M. R. (2006). Los hombres y las mujeres en México: Dos mundos distantes y complementarios ( $2^{\mathrm{a}}$ ed.). Estado de México, México; Universidad Autónoma del Estado de México.

Valdez, M. J. L. (1998). Las redes semánticas naturales. Usos y aplicaciones en Psicología Social. México: Universidad Autónoma del Estado de México.

Valdez-Medina, J. L. (2009). Teoría de la paz o equilibrio. Distrito Federal, México: EDAMEX.

Recibido el 01 de septiembre de 2015

Revisado el 14 de diciembre de 2015

Aceptado el 04 de marzo de 2016 